\title{
Fine needle aspiration cytology of lymph nodes in breast cancer follow-up is a feasible alternative to watchful waiting and to histology
}

Matthias Hammon ${ }^{1 *+}$, Peter Dankerl ${ }^{1+}$, Rolf Janka ${ }^{1}$, David L. Wachter ${ }^{2}$, Arndt Hartmann ${ }^{2}$, Rüdiger Schulz-Wendtland ${ }^{1}$, Michael Uder ${ }^{1}$ and Evelyn Wenkel ${ }^{1}$

\begin{abstract}
Background: Early detection of loco-regional breast cancer recurrence improves patients' overall survival, as treatment can be initiated or active treatment can be changed. If a suspicious lymph node is diagnosed during a follow-up exam, surgical excision is often performed. The aim of this study was to evaluate the diagnostic performance of the minor invasive ultrasound-guided fine-needle aspiration cytology (FNAC) in sonomorphologically suspicious lymph nodes in breast cancer follow-up.

Methods: Between April 2010 and November 2012, we performed ultrasound-guided FNAC in 38 sonographically suspicious lymph nodes of 37 breast cancer follow-up patients. Cytological specimens were evaluated if the sample material was sufficient for diagnosis and if they contained cancer cells. Patients with negative cytology were followed up clinically and sonographically. To evaluate the diagnostic performance we calculated sensitivity, specificity, positive predictive value (PPV) and negative predictive value (NPV) for physical examination, the different sonomorphological malignancy criteria and FNAC.
\end{abstract}

Results: In 36/38 (94.7 \%) lymph nodes, the pathologist had enough material to establish a final diagnosis; in 2/38 (5.3 \%) lymph nodes, the probe material was non-evaluable during cytology, these 2 were excluded from further statistical evaluation. Cytology revealed malignancy in 21 lymph nodes and showed no evidence for malignancy in 15 lymph nodes. There was no evidence for malignant disease in follow-up exams in the 15 cytologically benign lymph nodes with an average follow-up time of 3 years. The diagnostic performances of physical examination and FNAC were: Sensitivity 52/100 \%, specificity 88/100 \%, PPV 85/100 \%, NPV 60/100 \%, respectively.

Conclusions: Our preliminary results show that FNAC is a safe and fast diagnostic approach for the evaluation of suspicious lymph nodes in the follow-up of patients with breast cancer and, thus, together with follow-up represents a feasible alternative to surgery.

Keywords: Lymphatic, Metastasis, Breast, Ultrasound, Fine-needle aspiration cytology

\footnotetext{
* Correspondence: matthias.hammon@uk-erlangen.de

${ }^{\dagger}$ Equal contributors

'Department of Radiology, University Hospital Erlangen, Maximiliansplatz 1,

91054 Erlangen, Germany

Full list of author information is available at the end of the article
}

\section{Ciomed Central}

(c) 2015 Hammon et al. Open Access This article is distributed under the terms of the Creative Commons Attribution 4.0 International License (http://creativecommons.org/licenses/by/4.0/, which permits unrestricted use, distribution, and reproduction in any medium, provided you give appropriate credit to the original author(s) and the source, provide a link to the Creative Commons license, and indicate if changes were made. The Creative Commons Public Domain Dedication waiver (http://creativecommons.org/publicdomain/zero/1.0/) applies to the data made available in this article, unless otherwise stated. 


\section{Background}

Breast cancer is the most frequently diagnosed solid cancer in women and one of the leading causes of cancer deaths in the western world [1]. While screening mammography has led to the earlier detection of breast cancer [2], and guideline adherent therapy has improved overall and recurrence-free survival [3], detection of breast cancer recurrence remains difficult [4]. Data from meta-analysis and retrospective studies confirm that early detection of local recurrences resulted in significantly better survival as compared to late detected recurrences [5-7].

Regional lymph node recurrence is uncommon and has been reported in 1-3\% of patients with early stage breast cancer and in 1.7-15.9\% of patients with any stage of breast cancer [8]. The examinations performed to detect and assess lymph node recurrence should be reasonable regarding the patient's quality of life and time and cost-effectiveness [9]. Follow-up exams have to manage the difficult task of not inducing too much anxiety in patient with unnecessary exams or interventions and to minimize complications and costs [10].

Loco-regional recurrence includes recurrent disease in the diseased breast and the ipsilateral lymph nodes in the axillary, the supra- and infraclavicular and the internal mammary region. Regular follow-up exams are dependent on institutional preferences and include physical examination, mammography and sonography and, in unclear breast findings, magnetic resonance tomography $[11,12]$. In newly diagnosed breast cancer, axillary lymph node evaluation is usually performed preoperatively by clinical examination, sonography and lymph node resection. However, core needle biopsy and ultrasound-guided fine-needle aspiration cytology (FNAC) $[13,14]$ have become possible alternatives. In newly diagnosed breast cancer, it has already been shown that FNAC can achieve high accuracy, sensitivity and specificity [11, 15-20] to predict lymph node metastases. To the authors' knowledge, there are no prospective studies about lymph node sonography and FNAC in follow-up exams of breast cancer patients.

The aim of our study was to evaluate the diagnostic performance of fine-needle aspiration cytology of sonomorphologically suspicious lymph nodes in breast cancer follow-up.

\section{Methods}

\section{Ethics statement}

This single-centre investigation was approved by the institutional review board of the University Hospital Erlangen and all procedures were in accordance with the Helsinki Declaration. The need for informed consent was waived.

\section{Patients}

From April 2010 to November 2012, we performed more than 2500 follow-up exams in patients with breast cancer. Routine follow-up of patients included clinical examination and sonography of the breasts and the locoregional lymph node stations every 6 months for the first 3 years after surgery and then in yearly intervals. Mammography of the affected side was performed every 6 months for the first 3 years after surgery and then yearly; the non-affected breast was examined by mammography yearly. Patients who presented with sonomorphologically suspicious lymph nodes in the ipsi- or contralateral axillary or supra/infraclavicular region were eligible to receive FNAC. Prior to ultrasound-guided FNAC, written informed consent was obtained from each patient. During this time frame we performed ultrasound-guided FNAC in 59 sonographically suspicious lymph nodes of 58 patients. Of these, 38 FNAC probes from 37 patients were obtained from breast cancer follow-up patients and enrolled for further investigation. The remaining 21 suspicious lymph nodes of 21 patients were examined for non-breast cancer follow-up reasons and therefore not included in this study. Patients with positive cytology received recurrent stage adapted treatment. All patients with negative FNAC results were to follow up by sonography of the locoregional lymph nodes stations with special focus on the initially punctured lymph node within three months after the FNAC. If this evaluation revealed no change and no further suspicious findings, these patients should return to their regular follow-up examinations.

\section{Sonography}

Sonography was performed by a diagnostic breast specialist (E.W.) with 10 years of experience or by supervised residents experienced in breast diagnostic rotation with high-resolution sonography equipment $(15 \mathrm{MHz}$, electronic focus, linear-array transducer) (Siemens Acuson, Erlangen, Germany). A lymph node was defined as sonographically suspicious if one of the following characteristics was positive: size (longitudinal $>2 \mathrm{~cm}$; transverse $>1.5 \mathrm{~cm}$ ); shape (longitudinal/transversal ratio $<2$, round), loss of central fatty hilum, cortex (eccentric or thickened; > $3 \mathrm{~mm}$, as adopted by Oz et al. [17]) (Fig. 1). Additionally, all sonographically suspicious lymph nodes were measured in two planes. If one patient presented with more than one pathological lymph node in the same lymph drainage region, the most suspicious lymph node was selected for FNAC. If the suspicious nodes were in different topographical regions, one suspicious node in every region could receive FNAC.

\section{Fine-needle aspiration cytology (FNAC)}

All ultrasound-guided FNAC were performed by one breast specialist (E.W.). After sterile draping and sufficient 

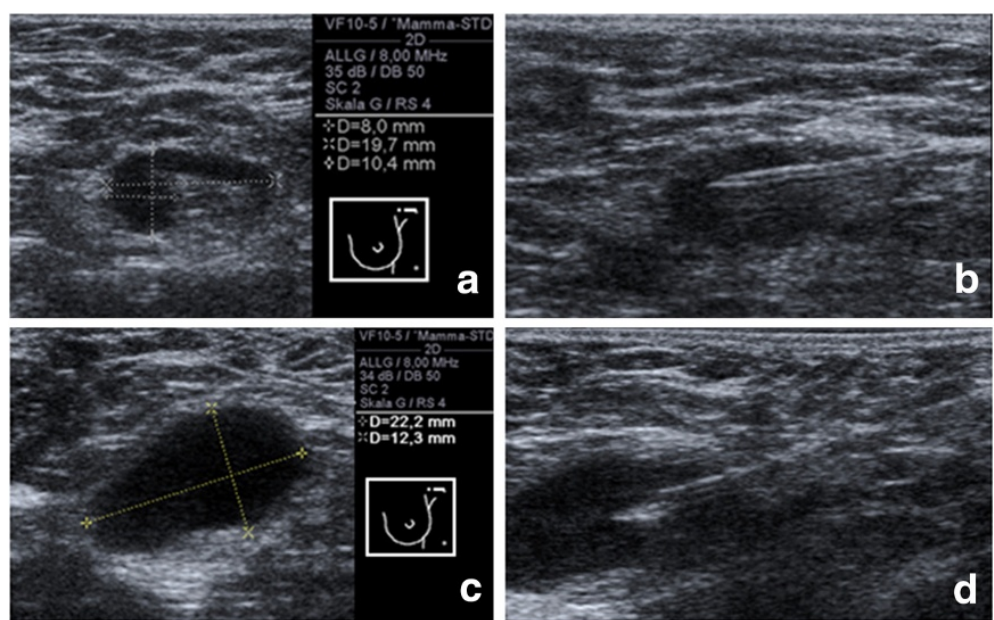

Fig. 1 Examples of sonographically suspicious axillary lymph nodes that received further fine needle aspiration cytology and cytological evaluation. a Lymph node presenting a normal size, a longitudinal/transversal ratio $<2$, a focal thickening of the cortex (8 mm) and an eccentric hilum. b Ultrasound-guided fine-needle aspiration cytology. c Lymph node with a round shape (longitudinal/transversal ratio $<2$ ), an indeterminate size and loss of fatty hilum. $\mathbf{d}$ Ultrasound-guided fine-needle aspiration cytology

disinfection, a 22-gauge needle attached to a 10-ml syringe was inserted into and withdrawn from the lymph node three times under aspiration while sonographically monitored. For suspiciously enlarged or shaped lymph nodes, the needle was directed into multiple areas of the cortex and/or hilum. For irregularly shaped lymph nodes, the needle was directed into the thickest or focally thickened area of the cortex and/or hilum (Fig. 1). Succeeding FNAC, the probe was sent to cytology. Following FNAC, patients were evaluated clinically for $60 \mathrm{~min}$ and 1 week after the procedure. Complications (bleeding, hematoma, infection, loss/increased sensation) were also documented.

\section{Cytological evaluation}

An expert in cytology and breast pathology [D.W.] with 9 years of experience processed and evaluated all FNAC material. In order to be able to perform immunocytochemical examinations in equivocal cases, we used cytocentrifugation and the cytoblock technique [21]. The FNAC cytology sample was concentrated by centrifugation (600 g for $10 \mathrm{~min}$ ). The supernatant was removed, and the pellet was resuspended in PreservCyt Solution (Cytyc Corporation, Marlborough, MA, USA). After sitting for $15 \mathrm{~min}$, the sample was run on a ThinPrep 2000 Processor (Cytyc Corporation, Marlborough, MassachusettsMA, USA). The resulting slide was stained with Papanicolaou stain, covered with a coverslip, and evaluated. For the cytoblock technique, part of the supernatant was fixed in $4 \%$ formaldehyde overnight. The next day the specimen was mixed with Histogel (Thermo Scientific, Waltham, MA, USA) and was allowed to harden. Subsequently the cytoblock was cut in $3 \mu \mathrm{m}$ slices and the slides were stained in Hematoxylin/Eosin. Specimens were interpreted as follows: insufficient, no cellular material other than blood; negative, only normal cellular components present or a specific benign diagnosis; suspicious or malignant, atypical cells suspicious or definite for malignancy present (Fig. 2).

\section{Statistical analysis}

Statistical analysis was performed using SPSS software version 21 (IBM, Armonk, New York, USA). Sensitivity, specificity, negative- (NPV) and positive-predictive value (PPV) were evaluated for physical examination, for the different sonomorphological malignancy criteria and for FNAC.

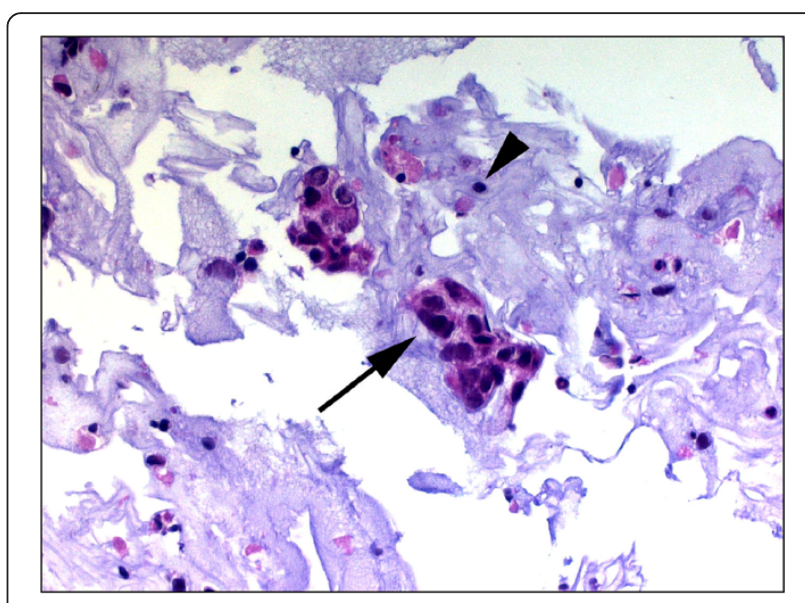

Fig. 2 Cell block cytology of an investigated lymph node displaying poorly differentiated breast cancer cells (arrow). The arrowhead indicates a lymphocyte 


\section{Results}

\section{Study population}

From April 2010 to November 2012, a total of 57 women and 1 man underwent ultrasound-guided fine-needle aspiration cytology of 59 axillary or supra- and infraclavicular lymph nodes. A total of 21 women with 21 cytologically evaluated lymph nodes were excluded because they were not breast cancer follow-up patients. Those women received FNAC for other reasons, like primary staging of breast cancer $(n=9)$, staging of other malignancies $(n=5)$ or primarily unknown reasons for lymph node enlargement ( $n=7$; mastitis, infection). Therefore, FNAC was performed on 38 lymph nodes in 37 follow-up patients for breast cancer. The 37 patients consisted of 36 women and one man with a mean age of $57.0 \pm 12.8$ years. A total of 19 FNAC were taken in the ipsilateral axilla, 14 in the contralateral axilla, 2 in the ipsilateral supraclavicular region, 1 in the contralateral supraclavicular region, 2 in the ipsilateral infraclavicular region and none in the contralateral infraclavicular region (Table 1). Follow-up time from primary diagnosis of breast cancer to the diagnosis of a sonomorphologically suspicious lymph node ranged from one month to 26 years and 6 months (Table 1).

\section{Physical examination}

A total of 13/38 sonographically suspicious lymph nodes were additionally identified by palpation, of which 7 were located in the ipsilateral axilla, 5 in the contralateral axilla and 1 in the contralateral supraclavicular. A total of 11 of these 13 lymph nodes demonstrated a malignant cytology in FNAC. A total of 10 metastatic lymph nodes were not suspicious upon palpation, of which 6 were located in the ipsilateral and 4 in the contralateral axilla. This corresponds to a sensitivity of $52.4 \%$ regarding palpation, a specificity of $88.2 \%$, a positive predictive value (PPV) of $84.6 \%$ and a negative predictive value (NPV) of $60 \%$.

\section{Sonography}

Sonomorphological characteristics of the lymph nodes, which were further assessed with fine-needle aspiration cytology, are shown in Table 2. All 59 lymph nodes showed at least one sonomophological pathological feature. The diagnostic performance of each individual sonomorphological malignancy criterion for lymph node evaluation is depicted in Table 3 . The sensitivity and the specificity of the different malignancy criteria ranged from $19-71 \%$ and from $29-88 \%$, the PPV from $42-75 \%$ and the NPV from $39-57 \%$.

\section{Fine-needle aspiration cytology and clinical consequences} FNAC evaluation revealed metastases in 21 lymph nodes in 21 patients, 20 women and 1 man. A total of 16 patients with positive FNAC received surgical resection of the suspicious lymph nodes. Specimen pathology confirmed malignancy in all of these cases. A total of 5 patients with positive cytology did not undergo surgery, and were instead treated with chemotherapy, antihormonal therapy and/or radiation therapy.

Probe material gathered by fine-needle aspiration was non-evaluable during cytology in 2 lymph nodes (5.3 \%) of two different patients because insufficient cell material was obtained. One was located in the ipsilateral and one in the contralateral axilla. Both patients with nondiagnostic cytological findings received successive lymph node resection of the initially biopsied lymph nodes. Specimen pathology did not reveal malignancy in either lymph node. FNAC did not indicate malignancy in 15 lymph nodes from 14 patients (Table 1).

The follow-up group consisted of 16 patients with 17 evaluated lymph nodes including the ones with negative cytology and the 2 patients with benign results after resection. To date, none of these patients presented with lymph node recurrence in the cytologically evaluated sites. The follow-up time of the patients with negative cytology or

Table 1 Lymph node and patient characteristics dependent on the fine-needle aspiration cytology result

\begin{tabular}{|c|c|c|c|}
\hline & Malignant & Benign & Non-evaluable \\
\hline Lymph nodes ( $n=38$ in 37 patients) & 21 & 15 & 2 \\
\hline Mean age in years (range) & $57(38-72)$ & $56(30-82)$ & $65(65-65)$ \\
\hline Lymph node palpable in clinical exam & 11 & 2 & 0 \\
\hline $\begin{array}{l}\text { Mean interval from primary diagnosis to ultrasound-guided } \\
\text { fine-needle aspiration cytology (range) }\end{array}$ & $4 y 9 m(1 m-13 y 7 m)$ & $7 y 5 m(5 m-26 y 6 m)$ & $4 y 6 m(2 y 3 m-6 y 9 m)$ \\
\hline \multicolumn{4}{|l|}{ Lymph node localisation } \\
\hline - axillar ipsilateral & 12 & 6 & 1 \\
\hline - axillar contralateral & 7 & 6 & 1 \\
\hline - supraclavicular ipsilateral & 1 & 1 & 0 \\
\hline - supraclavicular contralateral & 1 & 0 & 0 \\
\hline • infraclavicular ipsilateral & 0 & 2 & 0 \\
\hline - infraclavicular contralateral & 0 & 0 & 0 \\
\hline
\end{tabular}


Table 2 Sonomorphological characteristics of the lymph nodes which were further assessed with fine-needle aspiration cytology

\begin{tabular}{|c|c|c|c|}
\hline & Metastasis $(n=21)$ & No metastasis $(n=15)$ & Non-evaluable cytological probe $(n=2)$ \\
\hline \multicolumn{4}{|c|}{ Lymph node size longitudinal } \\
\hline$>2.0 \mathrm{~cm}$ & 15 & 8 & 1 \\
\hline$<2.0 \mathrm{~cm}$ & 6 & 7 & 1 \\
\hline \multicolumn{4}{|c|}{ Lymph node size transversal } \\
\hline$>1.5 \mathrm{~cm}$ & 6 & 2 & 0 \\
\hline$<1.5 \mathrm{~cm}$ & 15 & 13 & 2 \\
\hline \multicolumn{4}{|c|}{ Lymph node shape (longitudinal/transversal ratio) } \\
\hline - round $(<2)$ & 14 & 13 & 0 \\
\hline - oval (>2) & 7 & 2 & 2 \\
\hline \multicolumn{4}{|l|}{ Hilum } \\
\hline - loss of fat & 16 & 10 & 2 \\
\hline - eccentric & 4 & 2 & 0 \\
\hline Thickened cortex & 5 & 6 & 1 \\
\hline
\end{tabular}

histology ranged from 22 months (1 year 10 months) to 53 months (4 years 5 months). Thus, FNAC demonstrated sensitivity and specificity of $100 \%$ of sonomorphologically suspicious lymph nodes excluding the two cytologically non-evaluable probes.

\section{Fine-needle aspiration cytology side-effects}

None of our patients demonstrated acute or delayed side effects from FNAC (bleeding, hematoma, infection, loss/ increased sensation).

\section{Discussion}

Follow-up exams in breast cancer patients have to manage a fine line between early detection of recurrence and overdiagnosis. Follow-up concepts include clinical examination and mammography [11, 22, 23]. Lymph node sonography is performed in most institutions only if lymph node stations are suspicious on palpation or more uncommonly on each follow-up appointment [11, 12, $23,24]$. MRI is usually recommended if the differentiation between scar and recurrent disease is necessary or in women at high risk for breast cancer [25]. If there is an unclear lymph node finding on clinical or imaging examination, the woman has the choice between invasive diagnostic procedures like core needle biopsy or lymph node dissection. On the other hand, the woman could choose short follow-up exams especially in unclear lymph node findings. This might induce unnecessary anxiety in the patient up to the next control date.

The minimally invasive procedures core needle biopsy $[13,26,27]$ and FNAC have already been successfully tested in lymph nodes of newly diagnosed breast cancer [13, 14, 20, 28-31]. For core needle biopsies, a technique adapted to the axillary anatomy is recommended to minimize side effects [26]. A meta-analysis of data from 21 studies and 4313 patients showed that the pre-operative ultrasound of

Table 3 Diagnostic performance of physical examination, different sonomorphological malignancy criteria and fine-needle aspiration cytology for lymph node evaluation in breast cancer follow-up

\begin{tabular}{|c|c|c|c|c|}
\hline & Sensitivity & Specificity & Positive predictive value & Negative predictive value \\
\hline Physical examination & $52.4 \%$ & $88.2 \%$ & $84.6 \%$ & $60 \%$ \\
\hline \multicolumn{5}{|l|}{ Sonomorphological malignancy criteria } \\
\hline \multicolumn{5}{|l|}{ Lymph node enlargement: } \\
\hline - longitudinal > $2.0 \mathrm{~cm}$ & $71.4 \%$ & $47.1 \%$ & $62.5 \%$ & $57.1 \%$ \\
\hline - transversal > $1.5 \mathrm{~cm}$ & $28.6 \%$ & $88.2 \%$ & $75 \%$ & $50 \%$ \\
\hline Round shape (longitudinal/transversal ratio $<2$ ) & $66.7 \%$ & $23.5 \%$ & $51.8 \%$ & $36.4 \%$ \\
\hline Loss of fatty hilum & $76.2 \%$ & $29.4 \%$ & $57.1 \%$ & $50 \%$ \\
\hline Eccentric hilum & $19 \%$ & $88.2 \%$ & $66.7 \%$ & $46.9 \%$ \\
\hline Thickened cortex & $23.8 \%$ & $58.8 \%$ & $41.7 \%$ & $38.5 \%$ \\
\hline Fine-needle aspiration cytology ${ }^{a}$ & $100 \%$ & $100 \%$ & $100 \%$ & $100 \%$ \\
\hline
\end{tabular}

a excluding the two non-evaluable cytological probes 
axillary lymph nodes in primary breast cancer resulted in a median sensitivity of $61.4 \%$ (interquartile range 51.2$79.4 \%$ ) and a median specificity of $82 \%$ (interquartile range 76.9-89 \%) [32]. In a subset of 1733 patients in the same meta-analysis, the median sensitivity of ultrasound needle biopsy (results for core biopsy and FNAC) was $79.4 \%$ (interquartile range $68.3-88.9 \%$ ) and the median specificity was $100 \%$ (interquartile range 100-100 \%) [32]. No explicit difference was found in this meta-analysis whether FNAC or core biopsies were performed. In a meta-analysis of 20 studies with 1371 subjects, the performance of FNAC for lymph node metastases in primary breast cancers resulted in a pooled sensitivity of $66 \%$ (95\% CI was 64-69 \%) and a specificity of $98 \%$ (95\% CI was 98-99\%) [14]. As presented the diagnostic performance, especially the sensitivity of FNAC in post-operative breast cancer follow-up is much higher than in the preoperative staging of breast cancer patients. We believe that this is due to the different workflows and evaluation processes. In preoperative staging FNAC has been performed even if no lymph node was sonomorphologically suspicious on a random lymph node, in order to rule out metastases. However, if micrometastases had been present and coincidentally FNAC had been performed on a lymph node from a different draining system, these metastases could have not been detected by FNAC. The by us presented workflow eliminates the uncertainty of which lymph node to pick for FNAC, as the probed lymph node had to be newly detected and suspicious (either by physical examination or sonography), possibly explaining the perfect sensitivity. The excellent specificity of FNAC is in accordance to previous work [14], especially when performed by an experienced cytologist.

As in the pre-surgical studies, we show that FNAC is also reliable in lymph node stations that have already been tested. FNAC demonstrated sensitivity and specificity of $100 \%$ during the assessment of sonomorphologically suspicious lymph nodes when excluding two cytologically non-evaluable probes. Regarding cost-effectiveness, FNAC is proposed to be routinely included in pre-operative evaluation of lymph nodes [10, 13, 32, 33]. With the introduction of FNAC in suspicious lymph nodes in women who were examined during follow-up, we wanted to offer the least invasive tissue sampling method with a justifiable cost-benefit ratio. Furthermore, FNAC could be easily implemented in our follow-up procedure on the same day of the follow-up exam without major side effects. This is in accordance with various studies investigating FNAC in the primary diagnosis and staging of breast cancer $[10,16-18,20]$.

A total of 2 of 38 performed FNAC probe material gathered by fine-needle aspiration were non-evaluable during cytology because insufficient cell material was obtained. Both lymph nodes were completely resected and did not reveal metastatic infiltration in specimen pathology. All 15 patients demonstrating a negative FNAC were followed up clinically. Post-interventional examinations were performed one week (physical examination) and 3 months (physical examination, ultrasonography) after FNAC in addition to the routine follow-up protocol. During our surveillance period over 22-53 months, none of the 15 patients developed lymph node recurrence in the lymph region in which FNAC was performed. In our study, no complications were observed in any of the patients who underwent FNAC, which again demonstrates the safety of FNAC [15-18, 26, 33].

Clinical examination of lymph nodes in newly diagnosed breast cancer is already difficult because large reactive lymph nodes can be misleading and small metastatic lymph nodes are not palpable [14]. Treated lymph node stations are even more difficult to examine because of possible post-treatment side effects like scarring or hematoma. This follows from our evaluation, as clinical examination was suspicious only in $11 / 21$ malignant lymph nodes.

Though routine clinical examination is highly recommended in all follow-up programs, it is still unclear whether it contributes to relevant early recurrence detection. In a meta-analysis, there was no evidence that clinical examination contributes to survival advantage, as the majority of in-breast relapses are detected by patients or by mammography [34]. The rare axillary relapse is more often detected by clinical examination, as the axillary region is insufficiently evaluable on mammography and sonography was not performed in these studies.

Regarding sonography, all lymph nodes that were included in the study had at least one suspicious feature and only 21/37 lymph nodes were malignant. If evaluated separately, the different sonomorphological malignancy criteria reached a sensitivity and specificity ranging from $19-71 \%$ and from $29-88 \%$.

Limitations of the study are the small number of patients and the single institutional execution, however the initial experiences are promising.

Another limitation of the study is that the FNACnegative lymph nodes were not histologically verified and were only followed up. A follow-up time of at least 22 months seemed to be appropriate to define those lymph nodes as stable and consequently negative.

Sonography of lymph nodes after breast cancer treatment can be challenging. The sonographer has to differentiate between postsurgical alteration and chronic inflammation due to lymph edema or recurrent disease in the lymph nodes. The small number of patients seemed inadequate for the synthesis of further conclusions as to the value of the different ultrasound criteria.

We did not investigate the role of color Doppler ultrasound findings, which were described to be helpful in the 
differentiation of benign and malignant lymph nodes in primary breast cancer. Several studies have evaluated the vascularity of benign and malignant axillary lymph nodes, but the results were not consistent across the different studies, especially for lymph nodes smaller than $1 \mathrm{~cm}$ [35-37]. Recently, elastography was introduced as an additional feature in sonography of breast disease. In a prospective study of 104 axillary lymph nodes, strain elastography showed no improvement in diagnostic accuracy [38].

To date, it remains unclear which follow-up strategy is best for women to detect early recurrent disease and to minimize anxiety due to medical appointments. Debate persists with respect to the timeframe, extent of clinical and imaging examinations. Yearly mammography and clinical examination are the minimum guideline recommendations [11, 12, 22, 39]. Individual risk adapted follow-up examinations in future guidelines designed for optimal disease management are proposed $[39,40]$. Based on the results of this study, we can recommend FNAC as an additional diagnostic step regardless of the modality of a suspicious lymph node diagnosed during follow-up exams.

\section{Conclusions}

FNAC is a safe and reliable diagnostic tool for the evaluation of suspicious lymph nodes in breast cancer followup. It distinctly improves diagnostic performance of the evaluation of suspicious lymph nodes compared to clinical examination and an analysis based on sonomorphological malignancy criteria alone.

\section{Ethical approval and consent}

This single-centre investigation was approved by the institutional review board of the University Hospital Erlangen and all procedures were in accordance with the Helsinki Declaration. The need for informed consent was waived.

\section{Abbreviations}

FNAC: fine-needle aspiration cytology; NPV: negative predictive value; PPV: positive predictive value.

\section{Competing interests}

The authors declare that they have no competing interests.

\begin{abstract}
Authors' contributions
PD designed the study, carried out experimental work and drafted this manuscript; $\mathrm{MH}$ designed the study, carried out experimental work and drafted this manuscript; RJ helped in study design and drafting this manuscript; RSW helped in drafting of this manuscript; DLW carried out the cytological analysis; $\mathrm{AH}$ contributed to the cytological analysis and helped in study design; MU participated in the design of the study; EW performed the fine needle aspirations and performed a critical review of drafts of the manuscript. All authors read and approved final manuscript.
\end{abstract}

\section{Acknowledgements}

We thank our patients.

There was no funding for this study.

\section{Author details}

'Department of Radiology, University Hospital Erlangen, Maximiliansplatz 1, 91054 Erlangen, Germany. 'Department of Pathology, University Hospital Erlangen, Erlangen, Germany.

Received: 12 May 2015 Accepted: 20 November 2015

Published online: 03 December 2015

\section{References}

1. Siegel R, Naishadham D, Jemal A. Cancer statistics, 2012. CA Cancer J Clin. 2012;62:10-29.

2. Bleyer A, Welch $H G$. Effect of three decades of screening mammography on breast-cancer incidence. N Engl J Med. 2012;367:1998-2005.

3. Wöckel A, Kurzeder C, Geyer V, Novasphenny I, Wolters R, Wischnewsky M, et al. Effects of guideline adherence in primary breast cancer-a 5-year multi-center cohort study of 3976 patients. Breast. 2010;19:120-7.

4. Rojas MP, Telaro E, Russo A, Moschetti I, Coe L, Fossati R, et al. Follow-up strategies for women treated for early breast cancer. Cochrane Database Syst Rev. 2000; CD001768.

5. Lu WL, Jansen L, Post WJ, Bonnema J, Van de Velde JC, De Bock GH. Impact on survival of early detection of isolated breast recurrences after the primary treatment for breast cancer: a meta-analysis. Breast Cancer Res Treat. 2009;114:403-12.

6. Brooks JP, Danforth DN, Albert P, Sciuto LC, Smith SL, Camphausen KA, et al. Early ipsilateral breast tumor recurrences after breast conservation affect survival: an analysis of the National Cancer Institute randomized trial. Int J Radiat Oncol Biol Phys. 2005;62:785-9.

7. Arriagada R, Rutqvist LE, Mattsson A, Kramar A, Rotstein S. Adequate locoregional treatment for early breast cancer may prevent secondary dissemination. J Clin Oncol. 1995;13:2869-78.

8. Moon HJ, Kim MJ, Kim EK, Park BW, Youk JH, Kwak JY, et al. US surveillance of regional lymph node recurrence after breast cancer surgery. Radiology. 2009;252:673-81.

9. de Bock GH, Bonnema J, van der Hage J, Kievit J, van de Velde CJ. Effectiveness of routine visits and routine tests in detecting isolated locoregional recurrences after treatment for early-stage invasive breast cancer: a meta-analysis and systematic review. J Clin Oncol. 2004:22:4010-8.

10. Genta F, Zanon E, Camanni M, Deltetto F, Drogo M, Gallo R, et al. Cost/ accuracy ratio analysis in breast cancer patients undergoing ultrasound-guided fine-needle aspiration cytology, sentinel node biopsy, and frozen section of node. World J Surg. 2007;31:1155-63.

11. Khatcheressian JL, Hurley P, Bantug E, Esserman L, Grunfeld E, Halberg F, et al. Breast cancer follow-up and management after primary treatment: American Society of Clinical Oncology clinical practice guideline update. J Clin Oncol. 2013;31:961-5

12. Senkus E, Kyriakides S, Penault-Llorca F, Poortmans P, Thompson A, Zackrisson S, et al. Primary breast cancer: ESMO Clinical Practice Guidelines for diagnosis, treatment and follow-up. Ann Oncol. 2013;24 Suppl 6:vi7-23.

13. Ahn HS, Kim SM, Jang M, La Yun B, Kim SW, Kang E, et al. Comparison of sonography with sonographically guided fine-needle aspiration biopsy and core-needle biopsy for initial axillary staging of breast cancer. J Ultrasound Med. 2013;32:2177-84.

14. Wang XW, Xiong YH, Zen XQ, Lin HB, Liu QY. Diagnostic accuracy of ultrasonograph guided fine-needle aspiration cytologic in staging of axillary lymph node metastasis in breast cancer patients: a meta-analysis. Asian Pac J Cancer Prev. 2012;13:5517-23.

15. Gilani SM, Fathallah L, Al-Khafaji BM. Preoperative fine needle aspiration of axillary lymph nodes in breast cancer: clinical utility, diagnostic accuracy and potential pitfalls. Acta Cytol. 2014;58:248-54.

16. García Fernández A, Fraile M, Giménez N, Reñe A, Torras M, Canales L, et al. Use of axillary ultrasound, ultrasound-fine needle aspiration biopsy and magnetic resonance imaging in the preoperative triage of breast cancer patients considered for sentinel node biopsy. Ultrasound Med Biol. 2011; 37:16-22.

17. Oz A, Demirkazik FB, Akpinar MG, Soygur I, Baykal A, Onder SC, et al. Efficiency of ultrasound and ultrasound-guided fine needle aspiration cytology in preoperative assessment of axillary lymph node metastases in breast cancer. J Breast Cancer. 2012;15:211-7.

18. Gruber I, Hahn M, Fehm T, Hann von Weyhern C, Stäbler A, Winckelmann A, et al. Relevance and methods of interventional breast sonography in preoperative axillary lymph node staging. Ultraschall Med. 2012;33:337-43. 
19. Krishnamurthy S, Sneige N, Bedi DG, Edieken BS, Fornage BD, Kuerer HM, et al. Role of ultrasound-guided fine-needle aspiration of indeterminate and suspicious axillary lymph nodes in the initial staging of breast carcinoma. Cancer. 2002;95:982-8.

20. Mainiero MB, Cinelli CM, Koelliker SL, Graves TA, Chung MA. Axillary ultrasound and fine-needle aspiration in the preoperative evaluation of the breast cancer patient: an algorithm based on tumor size and lymph node appearance. AJR Am J Roentgenol. 2010;195:1261-7.

21. Kung IT, Chan SK, Lo ES. Application of the immunoperoxidase technique to cell block preparations from fine needle aspirates. Acta Cytol. 1990;34:297-303.

22. Puglisi F, Fontanella C, Numico G, Sini V, Evangelista L, Monetti F, et al. Follow-up of patients with early breast cancer: is it time to rewrite the story? Crit Rev Oncol Hematol. 2014;91:130-41.

23. Interdisziplinäre S3-Leitlinie für die Diagnostik, Therapie und Nachsorge des Mammakarzinoms. 2nd ed. Leitlinien Programm Onkologie. Vol. AWMF-RegisterNummer: 032 - 045OL. 2012, München: W. Zuckschwerdt Verlag GmbH.

24. NICE, National Institute for Health and Care Excellence. Early and locally advanced breast cancer. Diagnosis and treatment. NICE clinical guideline 80, 2014 (February 2009 last modified: July 2014).

25. Kuhl CK. Current status of breast MR imaging. Part 2. Clinical applications. Radiology. 2007;244:672-91.

26. Abe H, Schmidt RA, Kulkarni K, Sennett CA, Mueller JS, Newstead GM. Axillary lymph nodes suspicious for breast cancer metastasis: sampling with US-guided 14-gauge core-needle biopsy-clinical experience in 100 patients. Radiology. 2009;250:41-9.

27. Britton PD, Goud A, Godward S, Barter S, Freeman A, Gaskarth M, et al. Use of ultrasound-guided axillary node core biopsy in staging of early breast cancer. Eur Radiol. 2009;19:561-9.

28. Alkuwari E, Auger M. Accuracy of fine-needle aspiration cytology of axillary lymph nodes in breast cancer patients: a study of 115 cases with cytologichistologic correlation. Cancer. 2008;114:89-93.

29. Houssami N, Diepstraten SC, Cody 3rd HS, Turner RM, Sever AR. Clinical utility of ultrasound-needle biopsy for preoperative staging of the axilla in invasive breast cancer. Anticancer Res. 2014;34:1087-97.

30. Cools-Lartigue J, Sinclair A, Trabulsi N, Meguerditchian A, Mesurolle B, Fuhrer $\mathrm{R}$, et al. Preoperative axillary ultrasound and fine-needle aspiration biopsy in the diagnosis of axillary metastases in patients with breast cancer: predictors of accuracy and future implications. Ann Surg Oncol. 2013;20: 819-27.

31. Bonnema J, van Geel AN, van Ooijen B, Mali SP, Tjiam SL, Henzen-Logmans SC, et al. Ultrasound-guided aspiration biopsy for detection of nonpalpable axillary node metastases in breast cancer patients: new diagnostic method. World J Surg. 1997;21:270-4.

32. Houssami N, Ciatto S, Turner RM, Cody 3rd HS, Macaskill P. Preoperative ultrasound-guided needle biopsy of axillary nodes in invasive breast cancer: meta-analysis of its accuracy and utility in staging the axilla. Ann Surg. 2011; 254:243-51.

33. Cheng YS, Kuo SJ, Chen DR. Sparing sentinel node biopsy through axillary lymph node fine needle aspiration in primary breast cancers. World I Surg Oncol. 2013;11:296.

34. Montgomery DA, Krupa K, Cooke TG. Follow-up in breast cancer: does routine clinical examination improve outcome? A systematic review of the literature. Br J Cancer. 2007;97:1632-41.

35. Esen G, Gurses B, Yilmaz MH, Ilvan S, Ulus S, Celik V, et al. Gray scale and power Doppler US in the preoperative evaluation of axillary metastases in breast cancer patients with no palpable lymph nodes. Eur Radiol. 2005;15:1215-23.

36. Yang WT, Chang J, Metreweli C. Patients with breast cancer: differences in color Doppler flow and gray-scale US features of benign and malignant axillary lymph nodes. Radiology. 2000;215:568-73.

37. Tschammler A, Ott G, Schang T, Seelbach-Goebel B, Schwager K, Hahn D. Lymphadenopathy: differentiation of benign from malignant disease-color Doppler US assessment of intranodal angioarchitecture. Radiology. 1998;208: 117-23.
38. Park YM, Fornage BD, Benveniste AP, Fox PS, Bassett Jr RL, Yang WT. Strain elastography of abnormal axillary nodes in breast cancer patients does not improve diagnostic accuracy compared with conventional ultrasound alone. AJR Am J Roentgenol. 2014;203:1371-8.

39. Schneble EJ, Graham LJ, Shupe MP, Flynt FL, Banks KP, Kirkpatrick AD, et al. Future directions for the early detection of recurrent breast cancer. J Cancer. 2014;5:291-300.

40. Robertson C, Ragupathy SK, Boachie C, Fraser C, Heys SD, Maclennan G, et al. Surveillance mammography for detecting ipsilateral breast tumour recurrence and metachronous contralateral breast cancer: a systematic review. Eur Radiol. 2011;21:2484-91.

\section{Submit your next manuscript to BioMed Central and we will help you at every step:}

- We accept pre-submission inquiries

- Our selector tool helps you to find the most relevant journal

- We provide round the clock customer support

- Convenient online submission

- Thorough peer review

- Inclusion in PubMed and all major indexing services

- Maximum visibility for your research

Submit your manuscript at www.biomedcentral.com/submit
() Biomed Central 